\title{
Explicit stress integration of complex soil models
}

\author{
Jidong Zhao ${ }^{1, * \dagger}$, Daichao Sheng ${ }^{1}$, M. Rouainia ${ }^{2}$ and Scott W. Sloan ${ }^{1}$ \\ ${ }^{1}$ School of Engineering, University of Newcastle, NSW 2308, Australia \\ ${ }^{2}$ School of Civil Engineering and Geosciences, University of Newcastle, Newcastle upon Tyne NE1 7 RU, U.K.
}

\begin{abstract}
SUMMARY
In this paper, two complex critical-state models are implemented in a displacement finite element code. The two models are used for structured clays and sands, and are characterized by multiple yield surfaces, plastic yielding within the yield surface, and complex kinematic and isotropic hardening laws. The consistent tangent operators - which lead to a quadratic convergence when used in a fully implicit algorithm - are difficult to derive or may even not exist. The stress integration scheme used in this paper is based on the explicit Euler method with automatic substepping and error control. This scheme employs the classical elastoplastic stiffness matrix and requires only the first derivatives of the yield function and plastic potential. This explicit scheme is used to integrate the two complex critical-state models- the sub/superloading surfaces model (SSLSM) and the kinematic hardening structure model (KHSM). Various boundary-value problems are then analysed. The results for the two models are compared with each other, as well with those from standard Cam-clay models. Accuracy and efficiency of the scheme used for the complex models are also investigated. Copyright (C) 2005 John Wiley \& Sons, Ltd.
\end{abstract}

KEY WORDS: explicit stress integration; automatic substepping; critical state models; bounding surface; structured soil

\section{INTRODUCTION}

Critical-state soil models were first developed by Roscoe and his colleagues at Cambridge 30 years ago [1-4]. Since then, such models have been widely used in various geotechnical applications. Despite their popularity, the classic Cam-clay models are inadequate for addressing soil characteristics such as structures, anisotropy, small-strain behaviour, stiffness degradation during cyclic loading, and rate-dependent behaviour. Consequently, a variety of complex elastoplastic models have been proposed and they contain various modifications to the standard Cam-clay models to cover different soil types and loading behaviours [5-10]. In particular, the kinematic hardening structure model (KHSM) developed by Rouainia and Muir Wood [9] and the sub/super-loading surface model (SSLSM) proposed by Asaoka [10] are both capable of addressing natural soils with initial structures, and can simulate complicated soil response during cyclic loading. However, these models are mostly used to model the behaviour

\footnotetext{
*Correspondence to: J. Zhao, School of Engineering, University of Newcastle, NSW 2308, Australia.

${ }^{\dagger}$ E-mail: jidong.zhao@newcastle.edu.au
} 
of one single soil element and their applications to boundary-value problems are rather limited. The aim of the present study is to develop algorithmic and computational aspects of finite element implementation of the two models using an explicit stress-integration scheme.

Existing approaches for integrating stress-strain laws at Gauss points can be classified as 'explicit' schemes or 'implicit' schemes. Implicit algorithms based on the concepts of operator split and closest point projection or the so-called return mapping have been applied to a variety of computational geomechanics applications [11-22], while explicit schemes with substepping and error control have also been suggested in References [23-28] and applied to various geotechnical problems [7,29,30]. The advantages and disadvantages of the two classes of schemes are well-known and extensive comparisons between them have been made in literature (see, e.g. References [27,30-33]).

While both implicit and explicit schemes have been developed for the classic critical-state models [7,12,14-17,20], application of these schemes to more complex geomechanical models is limited. It is generally fair to state that highly non-linear complex constitutive models favours an explicit solution, because (i) the numerical solution to the local non-linear equations in an implicit scheme may not converge, (ii) the consistent tangent operator may be very difficult to derive so that the main advantage of the implicit schemes, i.e. the quadratic convergence of Newton iteration, is not guaranteed. In addition, certain geomechanical models, as the two model used in this paper, become so complex that it is very difficult to derive the second-order derivative of the plastic potential. Some exceptions of using implicit schemes for complex models include Bojar et al. [20] and Tamagnini et al. [21]. The former has used implicit scheme to solve an anisotropic bounding surface model. A loading surface that is homologous to the bounding surface in the strain space has to be introduced to handle the additional consistency condition on the bounding surface. Tamagnini et al. [21] used an implicit generalized backward Euler (GBE) method to treat an elastoplastic constitutive model for bonded geomaterials. Some assumptions such as material isotropy and hyperelastic behaviour had to be made to partially alleviate the drawbacks of implicit algorithms such as the requirement of computing the secondorder gradients of the plastic potential.

On the other hand, it is less cumbersome to use explicit integration schemes to solve complex constitutive models. In particular, the accuracy of these schemes, which is perceived to be their main drawback compared to the implicit schemes, can be overcome by using automatic substepping and error control. In this paper, the explicit scheme presented in References [26,27] is extended to solve two complex models. The two models KHSM and SSLSM both contain multiple yield surfaces and combined kinematic and isotropic hardening, invoked by the introduction of an initial structure and the bounding surfaces. The explicit scheme is reformulated to accommodate additional internal variables and hardening laws. The consistency conditions on the bounding surfaces are translated to the current loading surfaces. No addition measure is required to treat the consistency associated with the bounding surface. An automatic load stepping scheme is used to solve the global system of Equations [28,29], which ensures that the small-strain non-linearity in the KHSM and SSLSM is captured with accuracy and efficiency.

This paper is organized as follows. First, the KHSM and the SSLSM are briefly described and compared. Secondly, a general procedure for integrating stress-strain laws with both kinematic and isotropic hardening and more than one hardening parameters is presented. Thirdly, the two constitutive models are used to analyse triaxial compression tests under drained and footing conditions. The results of the two models are compared, as well as those from standard 
Cam-clay models. The performance of the explicit substepping integration scheme on KHSM and SSLSM is evaluated. Finally, the conclusions of the study are summarized.

\section{TWO COMPLEX SOIL MODELS}

In this section, the KHSM (Rouainia and Muir Wood [9]) and the SSLSM (Asaoka [10]) are briefly described. Slight modifications have been made to the formulation of each of the models to facilitate comparison. Both the KHSM and SSLSM were developed to account for initial structures, small strain stiffness, stiffness degradation with strain history, and hysteretic response in cyclic loading. In the KHSM, this was done by adding a structural surface into the kinematic hardening model developed by Al-Tabbaa and Muir Wood [34]; while in the SSLSM, an extra superloading surface was introduced into the subloading surface model proposed by Hashiguchi [35]. Concepts of bounding-surface plasticity [36] are used in both models. As a result, there are three surfaces of elliptical shape as in the MCC model in both models - the reference surface, the bubble surface, and the structural surface for the KHSM (see Figure 1(a) in the plane of $p^{\prime}-q$ ) and the normal yield surface, the subloading surface, and the superloading surface for the SSLSM (see Figure 1(b)). The small elastic region is confined by a small kinematic hardening bubble in the KHSM and the subloading surface in the SSLSM, respectively. The current stress state is always located within or on the bubble/subloading surface. Any stress path moving beyond the initial boundary of the bubble/subloading surface causes plastic deformation and evolution of all yield surfaces. The KHSM was initially presented in a form that can account for the initial anisotropy in soils by presenting a structure surface not passing through the origin. For simplicity, this feature is neglected here-with both the structure surface and the reference surface being assumed to pass through the origin. Consequently, it has a formulation similar to the model proposed by Muir Wood [6]. In the SSLSM, the three yield surfaces are all supposed to pass through the origin.

In both models, associated flow is assumed. The yield functions and corresponding plastic potentials for the yield surfaces in the two models are presented in Table I. In Table I, $p^{\prime}$ and $\mathbf{s}$ are the mean pressure and the deviatoric stress, respectively, given by $p^{\prime}=\operatorname{tr}[\boldsymbol{\sigma}] / 3, \mathbf{s}=\boldsymbol{\sigma}-p \mathbf{I}$, where $\mathbf{I}$ is the second-order identity tensor and $\operatorname{tr}[\cdot]$ is the trace operator of $[\cdot]$. For SSLSM,
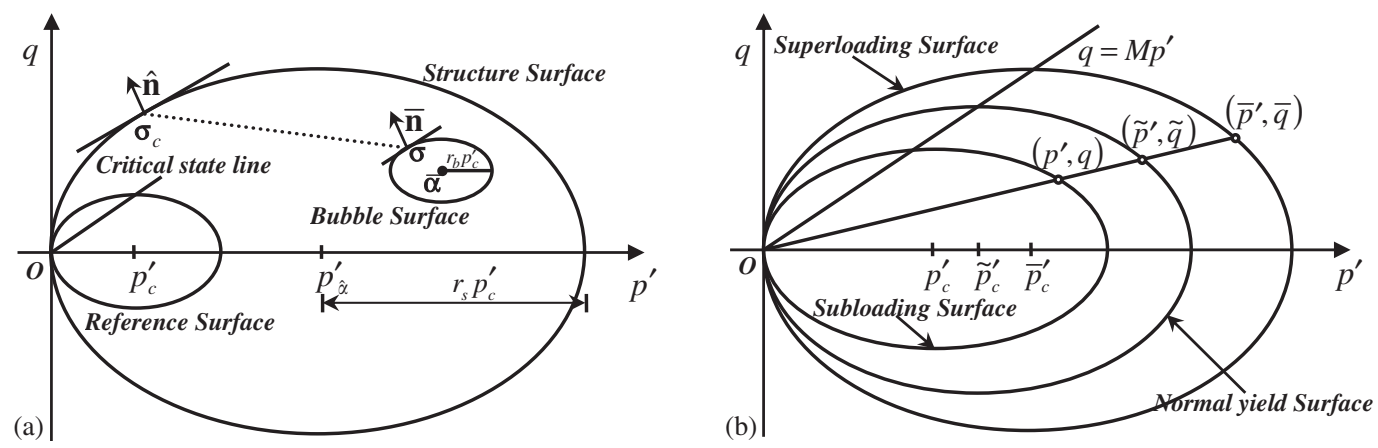

Figure 1. Illustration of yield surfaces for KHSM and SSLSM in the $p^{\prime}-q$ plane:

(a) KHSM [9]; (b) SSLSM [10]. 
Table I. Yield functions and corresponding plastic potentials for KHSM and SSLSM.

\begin{tabular}{lcc}
\hline Model & Yield surface & Yield function and plastic potential \\
\hline KHSM [9] & Reference surface & $f_{\mathrm{r}}=g_{\mathrm{r}}=\frac{\mathbf{s}: \mathbf{s}}{\left(M(\theta) p_{\mathrm{c}}^{\prime}\right)^{2}}+\left(\frac{p^{\prime}}{p_{\mathrm{c}}^{\prime}}-1\right)^{2}-1$ \\
Bubble surface & $f_{\mathrm{b}}=g_{\mathrm{b}}=\frac{\left(\mathbf{s}-\mathbf{s}_{\overline{\mathrm{a}}}\right):\left(\mathbf{s}-\mathbf{s}_{\overline{\mathrm{a}}}\right)}{\left(M(\theta) p_{\mathrm{c}}^{\prime}\right)^{2}}+\left(\frac{p^{\prime}-p_{\mathrm{a}}^{\prime}}{p_{\mathrm{c}}^{\prime}}\right)^{2}-R_{\mathrm{b}}^{2}$ \\
& Structure surface & $f_{\mathrm{s}}=g_{\mathrm{s}}=\frac{\mathbf{s}: \mathbf{s}}{\left(M(\theta) p_{\mathrm{c}}^{\prime}\right)^{2}}+\left(\frac{p^{\prime}}{p_{\mathrm{c}}^{\prime}}-R_{\mathrm{s}}\right)^{2}-R_{\mathrm{s}}^{2}$ \\
SSLSM [10] & Normal yield surface & $f_{\text {nor }}=g_{\text {nor }}=\frac{\tilde{\mathbf{s}}: \tilde{\mathbf{s}}}{\left(M(\theta) \tilde{p}_{\mathrm{c}}^{\prime}\right)^{2}}+\left(\frac{\tilde{p}^{\prime}}{\tilde{p}_{\mathrm{c}}^{\prime}}-1\right)^{2}-1$ \\
& Subloading surface & $f_{\text {sub }}=g_{\text {sub }}=\frac{\mathbf{s}: \mathbf{s}}{\left(M(\theta) p_{\mathrm{c}}^{\prime}\right)^{2}}+\left(\frac{p^{\prime}}{p_{\mathrm{c}}^{\prime}}-1\right)^{2}-1$ \\
& Superloading surface & $f_{\text {sup }}=g_{\text {sup }}=\frac{\overline{\mathbf{s}}: \mathbf{\mathbf { s }}}{\left(M(\theta) \bar{p}_{\mathrm{c}}^{\prime}\right)^{2}}+\left(\frac{\bar{p}^{\prime}}{\bar{p}_{\mathrm{c}}^{\prime}}-1\right)^{2}-1$ \\
\hline
\end{tabular}

$\left(p^{\prime}, \mathbf{s}\right),\left(\bar{p}^{\prime}, \overline{\mathbf{s}}\right)$ and $\left(\tilde{p}^{\prime}, \tilde{\mathbf{s}}\right)$ are the mean pressure and deviator stress at the subloading surface, superloading surface and normal-loading surface, respectively. Relations among them will be addressed afterwards. It should be noted that the slope of the critical state line (CSL) (designated by $M$ in the table) is expressed as a function of the Lode angle $\theta$, and determines the shape of the failure surface in the deviatoric plane. The following expression for $M$ is used in this paper:

$$
M(\theta)=M_{\max }\left(\frac{2 \alpha^{4}}{1+\alpha^{4}-\left(1-\alpha^{4}\right) \sin 3 \theta}\right)^{1 / 4}
$$

By setting the parameter $\alpha$ with $\alpha=(3-\sin \phi) /(3+\sin \phi)$, this yield surface coincides with the Mohr-Coulomb hexagon at all vertices in the deviatoric plane (where $\phi$ is the friction angle of the soil at critical state), while setting $\alpha=1$, recovering the Drucker-Prager compression circle. It should be noted that this surface is differentiable for all stress states and is convex (provided $\alpha \geqslant 0.6$ ). The variation of yield surface cone with respect to $\alpha$ in the deviatoric stress plane is depicted in Figure 2.

Other parameters in Table I should be noted in conjunction with Figure 1. For the KHSM, $\left(p_{\mathrm{c}}^{\prime}, 0\right),\left(p_{\hat{\alpha}}^{\prime}, 0\right)$, and $\left(p_{\bar{\alpha}}^{\prime}, \mathbf{s}_{\bar{\alpha}}\right)$ (instead of $\left.\left(p_{\bar{\alpha}}^{\prime}, q_{\bar{\alpha}}\right)\right)$ are the centres of the reference surface in a general $3 \mathrm{D}$ stress space, the structure surface, and the bubble surface in a general $3 \mathrm{D}$ stress space, respectively. $R_{\mathrm{s}}=p_{\hat{\alpha}}^{\prime} / p_{\mathrm{c}}^{\prime}$ is a parameter indicating the initial structure, whereas $R_{\mathrm{b}}$ is the size ratio of the bubble surface over the reference surface (and is assumed to be constant during loading). The KHSM uses the slopes of the normal compression line and swelling line, $\lambda^{*}$ and $k^{*}$, in the plane of $\ln v-\ln p^{\prime}$, which is slightly different from standard critical-state models. For the SSLSM, $\left(p_{\mathrm{c}}^{\prime}, 0\right),\left(\tilde{p}_{\mathrm{c}}^{\prime}, 0\right)$, and $\left(\bar{p}_{\mathrm{c}}^{\prime}, 0\right)$ are the respective centres of the three surfaces. Moreover, $\left(p^{\prime}, q\right),\left(\tilde{p}^{\prime}, \tilde{q}\right)$, and $\left(\bar{p}^{\prime}, \bar{q}\right)$ are the corresponding stresses on the subloading, normal Cam-clay, and superloading yield surfaces in the $p^{\prime}-q$ plane, respectively. The three stress states are related to 


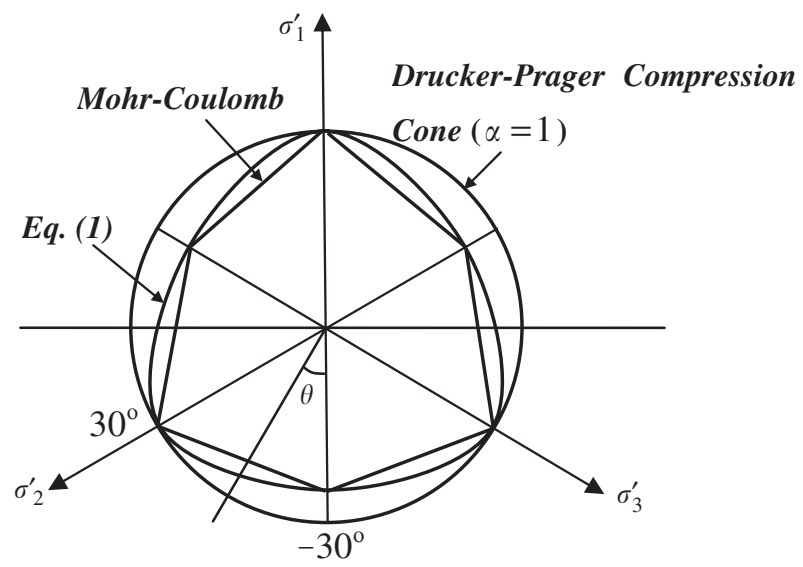

Figure 2. Variation of the yield surface with respect to $\alpha$ in the deviatoric stress plane.

one another by the following radial mapping rule:

$$
R=\frac{p^{\prime}}{\bar{p}^{\prime}}=\frac{q}{\bar{q}}, \quad R^{*}=\frac{\tilde{p}^{\prime}}{\bar{p}^{\prime}}=\frac{\tilde{q}}{\bar{q}}
$$

where $R$ and $R^{*}$ are similarity ratios for superloading/subloading surfaces and superloading/ normal surfaces, respectively. They act as constitutive hardening variables in the SSLSM. If $R=R^{*}=1.0$, the SSLSM will coincide with the modified Cam-clay model (MCCM). If in a more general 3D stress place, $\left(p^{\prime}, q\right),\left(\tilde{p}^{\prime}, \tilde{q}\right)$, and $\left(\bar{p}^{\prime}, \bar{q}\right)$ may be replaced by $\left(p^{\prime}, \mathbf{s}\right),\left(\bar{p}^{\prime}, \overline{\mathbf{s}}\right)$ and $\left(\tilde{p}^{\prime}, \tilde{\mathbf{s}}\right)$, respectively, where $\mathbf{s}, \overline{\mathbf{s}}$ and $\tilde{\mathbf{s}}$ have a same principal direction and yield a similar relations in norm form as (2) $R=\|\mathbf{s}\| /\|\overline{\mathbf{s}}\|, R^{*}=\|\tilde{\mathbf{s}}\| /\|\overline{\mathbf{s}}\|$.

The elastic moduli present themselves in the KHSM and the SSLSM in the following form:

$$
\begin{aligned}
& \text { KHSM: } K=\frac{\mathrm{d} p^{\prime}}{\mathrm{d} \varepsilon_{\mathrm{v}}^{\mathrm{e}}}=\frac{p^{\prime}}{k^{*}}, \quad G=\frac{3(1-2 \mu)}{2(1+\mu)} K \\
& \text { SSLSM: } K=\frac{\mathrm{d} p^{\prime}}{\mathrm{d} \varepsilon_{\mathrm{v}}^{\mathrm{e}}}=\frac{v p^{\prime}}{k}
\end{aligned}
$$

where $K$ and $G$ are the bulk modulus and the shear modulus, respectively. $\varepsilon_{\mathrm{v}}^{\mathrm{e}}$ denotes the elastic volumetric strain. $\mu$ is Poisson's ratio. $v=1+e$ is the specific volume, and $e$ is the void ratio.

The evolution of the plastic strain in the KHSM and the SSLSM is the same as in the standard Cam-clay model:

$$
\mathrm{d} \varepsilon_{i j}^{\mathrm{p}}=\mathrm{d} \gamma \frac{\partial g}{\partial \sigma_{i j}^{\prime}}
$$

where $\mathrm{d} \varepsilon_{i j}^{\mathrm{p}}$ denotes the plastic strain increment, $\mathrm{d} \gamma$ denotes the plastic multiplier. The following generalized form of hardening laws is supposed for both models:

$$
\mathrm{d} \mathbf{\kappa}=\mathrm{d} \gamma \mathbf{B}
$$

where $\boldsymbol{\kappa}$ denotes a vector of hardening variables and $\mathbf{B}$ is an intermediate vector used in the finite element formulation. In the KHSM the size of the bubble with respect to the reference 
surface can be assumed to be constant, so that $r_{\mathrm{b}}=$ const. In this case the KHSM has four hardening parameters: $p_{\mathrm{c}}^{\prime}, r_{\mathrm{s}}$, and the bubble centre $\left(p_{\bar{\alpha}}^{\prime}, \mathbf{s}_{\bar{\alpha}}\right)$. In the SSLSM, there are three hardening parameters: $p_{\mathrm{c}}^{\prime}, R$, and $R^{*}$. Thus the vectors $\mathbf{\kappa}$ and $\mathbf{B}$ in the two models have the following expressions:

$$
\begin{aligned}
& \text { KHSM: } \mathbf{\kappa}=\left\{\kappa_{1}, \kappa_{2}, \kappa_{3}, \kappa_{4}\right\}^{\mathrm{T}}=\left\{p_{\mathrm{c}}^{\prime}, R_{\mathrm{s}}, p_{\bar{\alpha}}^{\prime}, \mathbf{s}_{\bar{\alpha}}\right\}^{\mathrm{T}}, \quad B=\left\{B_{1}, B_{2}, B_{3}, B_{4}\right\}^{\mathrm{T}} \\
& \text { SSLSM: } \mathbf{\kappa}=\left\{\kappa_{1}, \kappa_{2}, \kappa_{3}\right\}^{\mathrm{T}}=\left\{p_{\mathrm{c}}^{\prime}, R, R^{*}\right\}^{\mathrm{T}}, \quad \mathbf{B}=\left\{B_{1}, B_{2}, B_{3}\right\}^{\mathrm{T}}
\end{aligned}
$$

Detailed expressions for the elements of B in the KHSM and the SSLSM may be referred to in Appendix A.

In the KHSM, the following geometric kinematic hardening mapping rule is used to ensure the movement of the bubble in a direction parallel to the line joining the current stress and the conjugate point on the bounding surface:

$$
\frac{\overline{\boldsymbol{\sigma}}_{\mathrm{c}}}{R_{\mathrm{s}}}=\frac{\overline{\boldsymbol{\sigma}}}{R_{\mathrm{b}}}
$$

where $\overline{\boldsymbol{\sigma}}_{\mathrm{c}}=\boldsymbol{\sigma}_{\mathrm{c}}-\hat{\boldsymbol{\alpha}}, \overline{\boldsymbol{\sigma}}=\boldsymbol{\sigma}-\overline{\boldsymbol{\alpha}}, \boldsymbol{\sigma}$ and $\boldsymbol{\sigma}_{\mathrm{c}}$ denotes the current stress on the bubble and its conjugate stress point (image stress) on the structure surface, respectively. In addition, the plastic modulus $H$ in the KHSM is assumed to depend on the Euclidean distance $b$ between the current stress and the conjugate stress (as illustrated in Figure 3).

It should be noted that both models can degenerate to the standard Cam-clay model by appropriate selection of model parameters. The MCC model will be recovered by the KHSM with $R_{\mathrm{b}}=R_{\mathrm{s}}=1.0$, and by the SSLSM with $R=R^{*}=1.0$. In addition, both models use the same the destructuration laws $R^{*}=1 / R_{\mathrm{s}}$, given $k=M a, \lambda^{*}=\lambda / v, k^{*}=k / v$ and under the same plastic deformation index (for example, $\varepsilon_{\mathrm{d}}$ in this paper), where $R_{\mathrm{s}}$ and $R^{*}$ are the destructuration index in the KHSM and SSLSM, respectively. However, the two models also exhibit obvious differences, in such aspects as the anisotropic nature of the model itself, the mapping rule, and the description of the reconsolidation process for soils. Details may be referred to in $[9,10]$.

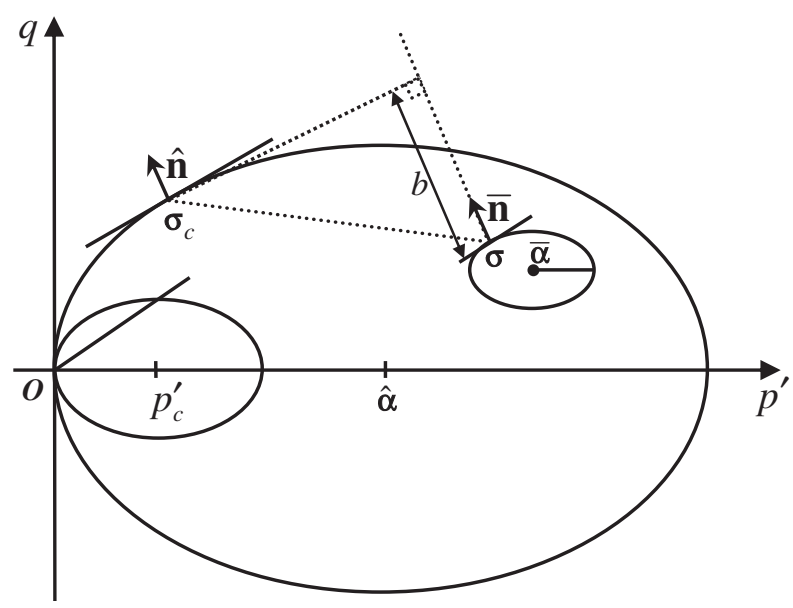

Figure 3. Illustration of the translation rule and normalized distance for KHSM. 


\section{EXPLICIT STRESS INTEGRATION}

\subsection{General formulation for finite element implementation}

During a typical elastoplastic finite element analysis, the following system of ordinary differential equations is to be solved:

$$
\begin{aligned}
& \dot{\boldsymbol{\sigma}}=\mathbf{D}_{\mathrm{ep}} \dot{\boldsymbol{\varepsilon}} \\
& \dot{\boldsymbol{\kappa}}=\dot{\gamma} \mathbf{B}
\end{aligned}
$$

where $\dot{\boldsymbol{\sigma}}$ and $\dot{\boldsymbol{\varepsilon}}$ are the rate of the stress and strain, respectively. $\dot{\boldsymbol{\kappa}}=\left\{\dot{\kappa}_{1}, \dot{\kappa}_{2}, \ldots, \dot{\kappa}_{n}\right\}^{\mathrm{T}}$ denotes the rate of the hardening parameter vector, and $\mathbf{B}^{\mathrm{T}}=\left\{\partial \kappa_{1} / \partial \gamma, \partial \kappa_{2} / \partial \gamma, \ldots, \partial \kappa_{n} / \partial \gamma\right\}$. The elastoplastic stiffness matrix and plastic multiplier are defined through

$$
\mathbf{D}_{\mathrm{ep}}=\mathbf{D}_{\mathrm{e}}-\frac{\mathbf{D}_{\mathrm{e}} \mathbf{b a}^{\mathrm{T}} \mathbf{D}_{\mathrm{e}}}{A+\mathbf{a}^{\mathrm{T}} \mathbf{D}_{\mathrm{e}} \mathbf{b}}, \quad \dot{\gamma}=\frac{\mathbf{a}^{\mathrm{T}} \mathbf{D}_{\mathrm{e}} \dot{\boldsymbol{\varepsilon}}}{A+\mathbf{a}^{\mathrm{T}} \mathbf{D}_{\mathrm{e}} \mathbf{b}}
$$

where $\mathbf{D}_{\mathrm{e}}$ is the elastic stiffness matrix. For the two models to be implemented, expressions in Equation (3) are used for $K$ and $G$. The generalized hardening modulus $A$ and the gradients of yield surface and plastic potential are defined by

$$
A=-\left(\frac{\partial f}{\partial \boldsymbol{\kappa}}\right)^{\mathrm{T}} \frac{\dot{\boldsymbol{\kappa}}}{\dot{\gamma}}, \quad \mathbf{a}=\frac{\partial f}{\partial \boldsymbol{\sigma}}, \quad \mathbf{b}=\frac{\partial g}{\partial \boldsymbol{\sigma}}
$$

The generalized hardening modulus $A$ in the KHSM has the following form:

$$
A=-\frac{\partial f_{\mathrm{b}}}{\partial p_{\mathrm{c}}^{\prime}} \frac{\partial p^{\prime}}{\partial \varepsilon_{\mathrm{v}}^{\mathrm{p}}} \frac{\partial f_{\mathrm{b}}}{\partial p^{\prime}}=\left(\frac{\partial f_{\mathrm{b}}}{\partial p_{\bar{\alpha}}^{\prime}} \frac{\partial p_{\bar{\alpha}}^{\prime}}{\partial \varepsilon_{\mathrm{d}}}+\frac{\partial f_{\mathrm{b}}}{\partial q_{\bar{\alpha}}} \frac{\partial q_{\bar{\alpha}}}{\partial \varepsilon_{\mathrm{d}}}\right) \sqrt{\left(1-A_{\mathrm{d}}\right)\left(\frac{\partial f_{\mathrm{b}}}{\partial p^{\prime}}\right)^{2}+A_{\mathrm{d}}\left(\frac{\partial f_{\mathrm{b}}}{\partial q}\right)^{2}}
$$

whereas in the SSLSM, it can be expressed as

$$
A=-\frac{\partial f_{\text {sub }}}{\partial p_{\mathrm{c}}^{\prime}} \frac{\partial p_{\mathrm{c}}^{\prime}}{\partial \tilde{p}_{\mathrm{c}}^{\prime}} \frac{\partial \tilde{p}_{\mathrm{c}}^{\prime}}{\partial \varepsilon_{\mathrm{v}}^{\mathrm{p}}} \frac{\partial f_{\text {sub }}}{\partial p^{\prime}}-\frac{\partial f_{\text {sub }}}{\partial p_{\mathrm{c}}^{\prime}}\left(\frac{\partial p_{\mathrm{c}}^{\prime}}{\partial R} \frac{\partial R}{\partial \varepsilon_{\mathrm{d}}}+\frac{\partial p_{\mathrm{c}}^{\prime}}{\partial R^{*}} \frac{\partial R^{*}}{\partial \varepsilon_{\mathrm{d}}}\right) \sqrt{\left(1-A_{\mathrm{d}}\right)\left(\frac{\partial f_{\text {sub }}}{\partial p^{\prime}}\right)^{2}+A_{\mathrm{d}}\left(\frac{\partial f_{\text {sub }}}{\partial q}\right)^{2}}
$$

The following pseudo-time, $T$, is defined to facilitate the integration of (8)

$$
T=\left(t-t_{0}\right) / \Delta t
$$

where $t_{0}$ is the time at the start of the load increment, $t_{0}+\Delta t$ is the time at the end of load increment, and $0 \leqslant T \leqslant 1$. Since $\mathrm{d} T / \mathrm{d} t=1 / \Delta t$, (9) may then be rewritten as

$$
\begin{aligned}
& \frac{\mathrm{d} \boldsymbol{\sigma}}{\mathrm{d} T}=\mathbf{D}_{\mathrm{ep}} \Delta \boldsymbol{\varepsilon}=\Delta \boldsymbol{\sigma}-\Delta \gamma \mathbf{D}_{\mathrm{e}} \mathbf{b} \\
& \frac{\mathrm{d} \boldsymbol{\kappa}}{\mathrm{d} T}=\dot{\gamma} \Delta t \mathbf{B}=\Delta \gamma \mathbf{B}
\end{aligned}
$$


where $\Delta \gamma=\mathbf{a}^{\mathrm{T}} \Delta \boldsymbol{\sigma}_{\mathrm{e}}\left(A+\mathbf{a}^{\mathrm{T}} \mathbf{D}_{\mathrm{e}} \mathbf{b}\right)$. Equations (13) and (14) define a classical initial value problem to be integrated over the pseudo-time interval $T=0$ to 1 .

\subsection{Explicit stress integration}

The explicit integration scheme with automatic substepping and error control [26,27] is used here to integrate the rate form of the stress-strain relations for the two models. A general procedure of this scheme include: (a) locating the yield surface intersection with the elastic trial stress path; (b) integrating the stress-strain relations using the modified Euler scheme with substepping and error control; and (c) correcting the yield surface drift if any. The procedures for implementing the KHSM and SSLSM closely follow that described in Reference [27] and only the necessary modifications are given below.

3.2.1. Yield surface intersection. An elastic trial stress increment $\Delta \boldsymbol{\sigma}_{\mathrm{e}}$ is first computed upon the imposed strain increments $\Delta \varepsilon$ :

$$
\Delta \boldsymbol{\sigma}_{\mathrm{e}}=\mathbf{D}_{\mathrm{e}} \Delta \boldsymbol{\varepsilon}
$$

For both the KHSM and SSLSM, the elastic part of the constitutive relation is non-linear. However, the incremental relation between the mean stress and the elastic volumetric strain can be integrated analytically to give the following secant elastic moduli:

$$
\begin{aligned}
& \operatorname{KHSM}: \bar{K}=\frac{p_{0}^{\prime}}{\Delta \varepsilon_{\mathrm{v}}^{\mathrm{e}}}\left(\exp \left(\Delta \varepsilon_{\mathrm{v}}^{\mathrm{e}} / k^{*}\right)-1\right) \\
& \text { SSLS: } \bar{K}=\frac{p_{0}^{\prime}}{\Delta \varepsilon_{\mathrm{v}}^{\mathrm{e}}}\left(\exp \left(v \Delta \varepsilon_{\mathrm{v}}^{\mathrm{e}} / k\right)-1\right)
\end{aligned}
$$

where $p_{0}^{\prime}$ is the effective mean stress at the start of the strain increment $\Delta \varepsilon_{\mathrm{v}}^{\mathrm{e}}$. Poisson's ratio is assumed to be constant when deriving the secant shear modulus $\bar{G}$. Accordingly, the secant elastic stiffness matrix $\overline{\mathbf{D}}_{\mathrm{e}}$ may be computed by $\bar{K}$ and $\bar{G}$, and (15) may be replaced by

$$
\Delta \overline{\boldsymbol{\sigma}}_{\mathrm{e}}=\overline{\mathbf{D}}_{\mathrm{e}} \Delta \boldsymbol{\varepsilon}
$$

where $\bar{K}$ and $\bar{G}$, and thus $\overline{\mathbf{D}}_{\mathrm{e}}$ are evaluated by the initial stress state $\boldsymbol{\sigma}_{0}$ and the total volumetric strain increments $\Delta \varepsilon_{\mathrm{v}}$. The obtained elastic trial stress increment $\Delta \overline{\boldsymbol{\sigma}}_{\mathrm{e}}$ can then be used to check if plastic yielding occurs. The exact yield condition, $f(\boldsymbol{\sigma}, \boldsymbol{\kappa})=0\left(f_{\mathrm{b}}\right.$ for the KHSM and $f_{\text {sub }}$ for the SSLSM, respectively), is approximated by an appropriate small tolerance FTOL (typically ranged from $10^{-9}$ to $\left.10^{-12}\right)$ via: $\mid f\left(\boldsymbol{\sigma}, \boldsymbol{\kappa} \mid \leqslant\right.$ FTOL. If $f\left(\boldsymbol{\sigma}_{0}, \boldsymbol{\kappa}_{0}\right)<-$ FTOL and $f\left(\boldsymbol{\sigma}_{0}+\Delta \overline{\boldsymbol{\sigma}}_{\mathrm{e}}, \boldsymbol{\kappa}_{0}\right)>+$ FTOL, an elastoplastic transition occurs, and an efficient and accurate algorithm such as the Pegasus intersection method as present in Reference [27] may be used to ascertain the fraction of $\Delta \boldsymbol{\varepsilon}$ that moves the stresses from $\boldsymbol{\sigma}_{0}$ to the stress state $\boldsymbol{\sigma}_{\text {int }}$ on the yield surface $\left(f_{\mathrm{b}}\right.$ for the KHSM and $f_{\text {sub }}$ for the SSLSM, respectively). The extra internal variables introduced by the bounding surface in the KHSM and the SSLSM will not evolve during this elastic trial process, as they are all assumed to be related with plastic deformation only (referred in Appendix A).

For every stress point on the current yield surface, there is an image (conjugate) stress point on the bounding surface. We further exam the change of the image stress point after locating the yield surface intersection. As an illustration, we choose the simple triaxial cases $\left(p^{\prime}, q\right)$ to be examined here, and it is easy to extend them to general 3D cases where the 
Lode angle is also considered. For the KHSM, once the yield surface intersection $\boldsymbol{\sigma}_{\text {int }}$ is found, the elastic incremental stress $\Delta \boldsymbol{\sigma}_{\mathrm{e}}=\boldsymbol{\sigma}_{\text {int }} \boldsymbol{\sigma}_{0}$ can be found exactly, using the secant elastic moduli and the portion of the strain increment. The stress state $\boldsymbol{\sigma}_{\text {int }}$ satisfies $f_{\mathrm{b}}\left(\boldsymbol{\sigma}_{0}+\Delta \boldsymbol{\sigma}_{\mathrm{e}}, \boldsymbol{\kappa}_{0}\right) \leqslant \mathrm{FTOL}$, which is

$$
f_{\mathrm{b}}=\left(\frac{\left(q_{0}+\Delta q_{\mathrm{e}}-q_{\bar{\alpha}_{0}}\right):\left(q_{0}+\Delta q_{\mathrm{e}}-q_{\bar{\alpha}_{0}}\right)}{M p_{\mathrm{c} 0}^{\prime}}\right)^{2}+\left(\frac{p_{0}^{\prime}+\Delta p_{\mathrm{e}}^{\prime}-p_{\bar{\alpha}_{0}}^{\prime}}{p_{\mathrm{c} 0}^{\prime}}\right)^{2}-R_{\mathrm{b}}^{2} \leqslant \text { FTOL }
$$

In view of Equation (7), the image stress point on the structural surface is now

$$
\boldsymbol{\sigma}_{\mathrm{c}}=\frac{R_{\mathrm{b}}}{R_{\mathrm{s}}}\left(\boldsymbol{\sigma}_{0}+\Delta \boldsymbol{\sigma}_{\mathrm{e}}-\overline{\boldsymbol{\alpha}}_{0}\right)+\hat{\boldsymbol{\alpha}}_{0}
$$

Substitution of (19) into the yield function of structural surface in Table I leads to

$$
f_{\mathrm{s}}=\left(\frac{\left[R_{\mathrm{b}}\left(q_{0}+\Delta q_{\mathrm{e}}-q_{\bar{\alpha}_{0}}\right) / R_{\mathrm{s}}\right]}{M(\theta) p_{\mathrm{c} 0}^{\prime}}\right)^{2}+\left(\frac{R_{\mathrm{b}}\left(p_{0}^{\prime}+\Delta p_{\mathrm{e}}^{\prime}-p_{\bar{\alpha}_{0}}^{\prime}\right) / R_{\mathrm{s}}+p_{\hat{\alpha}_{0}}^{\prime}}{p_{\mathrm{c} 0}^{\prime}}-R_{\mathrm{s} 0}\right)^{2}-R_{\mathrm{s} 0}^{2}
$$

Note that $R_{\mathrm{s} 0}=p_{\hat{\boldsymbol{\alpha}}_{0}}^{\prime} / p_{\mathrm{c} 0}^{\prime}$. Rearranging Equation (20) leads to

$$
f_{\mathrm{s}}=\frac{R_{\mathrm{b}}}{R_{\mathrm{s} 0}}\left(f_{\mathrm{b}}+R_{\mathrm{b}}^{2}\right)-R_{\mathrm{s} 0}^{2}=\frac{R_{\mathrm{b}}}{R_{\mathrm{s} 0}} f_{\mathrm{b}}+\frac{R_{\mathrm{b}}^{3}-R_{\mathrm{s} 0}^{3}}{R_{\mathrm{s} 0}}
$$

Recall that $R_{\mathrm{b}} / R_{\mathrm{s} 0} \leqslant 1$. We find that $f_{\mathrm{s}} \leqslant \mathrm{FTOL}$, or alternatively

$$
f_{\mathrm{s}}\left(\boldsymbol{\sigma}_{0}^{(\mathrm{s})}+\Delta \boldsymbol{\sigma}_{\mathrm{e}}^{(\mathrm{s})}, \boldsymbol{\kappa}_{0}\right) \leqslant \text { FTOL }
$$

Equation (22) indicates that the image stress point of $\boldsymbol{\sigma}_{\text {int }}$ is consistently within or on the structural surface. Following the same procedure, we can arrive at the same conclusion for SSLSM.

3.2.2. Modified Euler scheme with substepping. Once the portion of the given strain increment that causes plastic yielding is known, a set of stress increments and a set of increments of the hardening parameters can be computed using the forward Euler method, with all stressdependent quantities estimated at the current stress state. We then can update the stress state and hardening parameters according to the forward Euler solution. Using the updated stress state and the updated hardening parameter to estimate elastic stiffness and gradients of the yield surface and plastic potential, we can obtain another set of stress increments and hardening parameter increments, i.e. the modified Euler solution. The difference between the two sets of solutions can then be used as an error measure. The strain increment is subdivided if the error is larger than a prescribed tolerance. If the error is within the tolerance, the stress state and hardening parameters are then updated according to the modified Euler method. The substepping scheme used here largely follows that presented in Reference [27] for critical state models. The relative error in the stress solutions follows exactly that in Reference [27]. However, the relative error in the hardening parameters is computed as follows for the KHSM and 
SSLSM, respectively:

$$
\begin{aligned}
& \text { KHSM: } \operatorname{ERR}_{n}^{(\kappa)} \\
& =\sqrt{\frac{\left(\Delta p_{\mathrm{c} 2}^{\prime}-\Delta p_{\mathrm{c} 1}^{\prime}\right)^{2}}{\left(p_{\mathrm{c}}^{\prime}\right)^{2}}+\frac{\left(\Delta p_{\bar{\alpha} 2}^{\prime}-\Delta p_{\bar{\alpha} 1}^{\prime}\right)^{2}}{\left(p_{\bar{\alpha}}^{\prime}\right)^{2}}+\frac{\left(\Delta R_{\mathrm{s} 2}-\Delta R_{\mathrm{s} 1}\right)^{2}}{\left(R_{\mathrm{s}}\right)^{2}}+\frac{\left(\Delta \mathbf{s}_{\bar{\alpha} 2}-\Delta \mathbf{s}_{\bar{\alpha} 1}\right):\left(\Delta \mathbf{s}_{\bar{\alpha} 2}-\Delta \mathbf{s}_{\bar{\alpha} 1}\right)}{\mathbf{s}_{\bar{\alpha}}: \mathbf{s}_{\bar{\alpha}}}} \\
& \operatorname{SSLSM}: \operatorname{ERR}_{n}^{(\kappa)}=\sqrt{\frac{\left(\Delta p_{\mathrm{c} 2}^{\prime}-\Delta p_{\mathrm{c} 1}^{\prime}\right)^{2}}{\left(p_{\mathrm{c}}^{\prime}\right)^{2}}+\frac{\left(\Delta R_{2}-\Delta R_{1}\right)^{2}}{(R)^{2}}+\frac{\left(\Delta R_{2}^{*}-\Delta R_{1}^{*}\right)^{2}}{\left(R^{*}\right)^{2}}}
\end{aligned}
$$

where the subscript 2 stands for the second-order accurate solution obtained by the modified Euler method, the subscript 1 stands for the first-order accurate solution obtained by the forward Euler method, and all the denominators use the second-order accurate solutions. In Equation (23), we include the error in each hardening parameter, even though some parameters (such as $R_{\mathrm{S}}$ for the KHSM) do not explicitly appear in the current yield surface. The larger value between the stress error and the hardening parameter error is then used to subincrement the strain increment.

In the KHSM, the translation law in Equation (A1) should theoretically guarantee that the bubble is always inside the structure surface. In numerical computation, however, this is not always the case and the bubble might have drifted slightly outside the structure surface. Test runs show that this drift is sensitive to the parameters $b$ and $b_{\max }$ used in the translation law as well as the material parameters $B, k$, and $\psi$. This drift, if left uncorrected, can lead to some numerical instability. The correction of this drift is discussed below. The SSLSM is more robust in the sense that the subloading surface always lies within the superloading surface.

3.2.3. Correction of yield surface drift. The stress state at the end of a successful subincrement may slightly drift away from the current yield surface. It is generally recommended to correct this drift, using for example a consistent scheme as described in Reference [27]. Supposing that the uncorrected stresses and hardening parameters, denoted by $\boldsymbol{\sigma}_{0}$ and $\boldsymbol{\kappa}_{0}$, violate the current yield condition so that $\left|f\left(\boldsymbol{\sigma}_{0}, \boldsymbol{\kappa}_{0}\right)\right|>$ FTOL, we can impose a small change $\boldsymbol{\delta} \boldsymbol{\sigma}$ and a small stress change $\boldsymbol{\delta} \kappa$ to bring the stress point back to the yield function. One condition is that such changes should not cause any strain. From Equation (14), we have

$$
\boldsymbol{\delta} \boldsymbol{\sigma}=-\delta \gamma \mathbf{D}_{\mathrm{e}} \mathbf{b}_{0}, \quad \boldsymbol{\delta} \mathbf{\kappa}=\delta \gamma \mathbf{B}_{0}
$$

where $\mathbf{D}_{\mathrm{e}}, \mathbf{b}_{0}$ and $\mathbf{B}_{0}$ are computed at $\boldsymbol{\sigma}_{0}$ and $\boldsymbol{\kappa}_{0}$. Our goal is now to find the scalar $\delta \gamma$. We can expand the yield function around $\boldsymbol{\sigma}_{0}$ and $\boldsymbol{\kappa}_{0}$ using the first-order terms in the Taylor series:

$$
f\left(\boldsymbol{\sigma}_{0}+\delta \boldsymbol{\sigma}, \mathbf{\kappa}_{0}+\delta \mathbf{\kappa}\right)=f_{0}+\mathbf{a}_{0}^{\mathrm{T}} \delta \boldsymbol{\sigma}+\frac{\partial f}{\partial \mathbf{\kappa}} \delta \mathbf{\kappa}=0
$$

where $f_{0}=f\left(\boldsymbol{\sigma}_{0}, \boldsymbol{\kappa}_{0}\right)$, and $\mathbf{a}_{0}$ is evaluated at $\boldsymbol{\sigma}_{0}$. Substituting (24) into (25) leads to

$$
\delta \gamma=f_{0} /\left(A_{0}+\mathbf{a}_{0}^{\mathrm{T}} \mathbf{D}_{\mathrm{e}} \mathbf{b}_{0}\right)
$$


where $A_{0}$ is evaluated by Equations (11) and (12) for the KHSM and SSLSM, respectively, using $\boldsymbol{\sigma}_{0}$ and $\boldsymbol{\kappa}_{0}$ for the current yield surface. Once $\delta \gamma$ is obtained, corrections to $\boldsymbol{\sigma}$ and $\boldsymbol{\kappa}$ are readily computed by (24) and the stresses and hardening parameters are updated as follows:

$$
\boldsymbol{\sigma}=\boldsymbol{\sigma}_{0}+\delta \boldsymbol{\sigma}, \quad \boldsymbol{\kappa}=\boldsymbol{\kappa}_{0}+\delta \boldsymbol{\kappa}
$$

This consistent correction scheme may be applied repeatedly until $|f(\boldsymbol{\sigma}, \mathbf{\kappa})| \leqslant$ FTOL.

For the SSLSM, the yielding condition of the current yield surface $\left(f_{\text {sub }}\right)$, i.e. Equation (26), contains all the hardening parameters. Therefore, the bounding surface $\left(f_{\text {sup }}\right)$ is updated accordingly. For the KHSM, the current yield surface $\left(f_{\mathrm{b}}\right)$ does not contain the hardening parameter $R_{\mathrm{s}}$. Therefore, we can either leave $R_{\mathrm{s}}$ unchanged, or update it using the same scalar $\delta \gamma$ given by (26). In this study, we use the latter option when the bubble surface is inside the structural surface, to be consistent with other hardening parameters. If the bubble surface is slightly drifted outside the structural surface at the end of a subincrement, the structural surface is then corrected by adjusting $R_{\mathrm{s}}$ so that the current stress point is identical to the image point, i.e. $f_{\mathrm{s}}(\boldsymbol{\sigma}, \mathbf{\kappa})=0$.

\subsection{Global stiffness equation solver}

The global stiffness equations are solved by the load stepping scheme with automatic subincrementation and error control as presented in References [28,29]. Such a scheme is similar to the stress integration method. We first compute the tangential stiffness based on the current stresses and solve the stiffness equation to obtain the forward Euler solution of nodal displacements. We then update the stresses, recompute the stiffness and resolve the stiffness equation to obtain the modified Euler solution. The two sets of displacements naturally construct an error measure, which is then used to subdivide the load step if it is larger than a prescribed tolerance. The displacement tolerance is typically set to $10^{-3}-10^{-5}$ and is set to $10^{-4}$ in this paper.

The automatic load stepping scheme represents a linear incremental procedure to approximate the load-deformation behaviour of the system of ordinary differential equations, in contrast with the iterative procedures as typically represented by the Newton-Raphson method or the modified Newton-Raphson method. The iterative procedures present an advantage of satisfying equilibrium equations at the end of each converged time step. Quadratic convergence may be achieved if the consistent tangent stiffness operators are used. However, in case of strongly non-linear material behaviour, as here for the KHSM and SSLSM, the iterations may not converge and the algorithmic consistent tangent operators may even not exist. The load stepping scheme with automatic subincrementation and error control retain the advantage of using small load increment for incremental procedure when necessary, and at the same time, is capable of minimizing the drift from equilibrium by computing the residual forces at the end of each load increment and adding these to the applied forces for the next increment. These features make the automatic load stepping scheme suitable for solving the governing equations at the global level for soils characterized by models with complex mechanical behaviours as in the KHSM and SSLSM.

\section{VERIFICATION AND APPLICATION}

In this section, the two soil models and standard critical-state models are used to analyse drained triaxial compression tests and a rigid footing problem. Accuracy, efficiency and robustness of the explicit schemes used for the two models are also examined. 


\subsection{Drained triaxial compression tests}

For the following triaxial compression tests, a quarter of the cylindrical specimen of 0.5 unit in diameter and 1.0 unit in length is discretized into eight triangular six-noded elements. The loading process resembles that in a conventional triaxial compression test in that the radial stress is kept constant while a prescribed axial strain is imposed gradually. The units of soil and geometric parameters are not important as long as they are consistent with each other. Quasistatic displacement analysis is used for the computation. An initial isotropic stress field with $\sigma_{r 0}^{\prime}=\sigma_{a 0}^{\prime}=34.5$ is applied to the soil specimen. An axial strain of $50 \%$ is imposed in 100 coarse increments. Sensitivity studies of the over-consolidation ratio and the initial structure ratio are first conducted for both the KHSM and the SSLSM. The two models are then used to simulate the mechanical responses of two types of soils. Examination of the consistency of accuracy and efficiency of the schemes used is then carried out for the KHSM.

4.1.1. Sensitivity studies of $O C R$ and initial structure ratio. Model parameters for the KHSM and SSLSM used in the sensitivity study are as presented in Table II. A lightly structured soil with OCR varying between 1.5 and 9 is modelled by the KHSM and the SSLSM, respectively, and the predicted stress-strain curves are presented in Figure 4(a) and (b). The initial structures in the KHSM and the SSLSM are set by $R_{\mathrm{S}}=1.5$ and $R^{*}=0.67$, respectively. Note that $R_{\mathrm{S}}$ in the KHSM is equivalent to $1 / R^{*}$ in the SSLSM. The effects of initial structure on the model response are investigated by considering normally consolidated soils. As can be seen, the shape of the stress-strain curves obtained by the two models is similar with each other. Both models predict higher peak strength with a larger OCR, and a flatter curve with a smaller OCR. The peak shear strengths for the KHSM appear at about 2.5-4.5\% axial strain, with a larger OCR causing a slightly lagged peak response. For the SSLSM, the peak strengths occur at axial strains between 3 and $5 \%$, with a smaller OCR causing a slightly lagged response.

Three different initial structures are assumed for both models (in the KHSM $R_{\mathrm{S}}=1,5,10$ and in the SSLSM $R^{*}=1.0,0.2,0.1$, respectively), and the predicted stress-strain curves are shown in Figure 4(c) and (d). As is shown, the KHSM generally predicts that the soil with an initial structure can generally sustain a larger shear stress, and the stress-strain curves for $R_{\mathrm{S}}=5$ and 10 actually display a peak shear strength. In contrast to the KHSM, the SSLSM predicts that the soil with an initial structure shows a smaller shear stress than the remoulded soil, and there is no obvious peak shear strength in the strain-stress curves with a large initial structure. However, this does not necessarily imply that the existence of initial structure will degrade the strength of the soil. On the contrary, according to Asaoka et al. [37], for the same soil, the higher

Table II. Model parameter selection for KHSM and SSLSM for the sensitivity studies.

\begin{tabular}{lcc}
\hline Models & KHSM & SSLSM \\
\hline Compression index & $\lambda^{*}=0.05$ & $\lambda=0.05$ \\
Swelling index & $k^{*}=0.035$ & $k^{*}=0.035$ \\
Poisson's ratio & \multicolumn{1}{c}{$\mu=0.3$} & \\
Frictional angle & \multicolumn{2}{c}{$\phi=35.3^{\circ}$} \\
Slope of CSL & \multicolumn{2}{c}{$M_{\max }=1.43$} \\
Other specific & $\omega=4.0, B=1.98, \psi=1.5$, & $m=0.127, a=0.092$ \\
parameters & $R_{\mathrm{b}}=0.1, A_{\mathrm{d}}=0.95$ & $A_{\mathrm{d}}=0.95, e_{0}=0.82$ \\
\hline
\end{tabular}



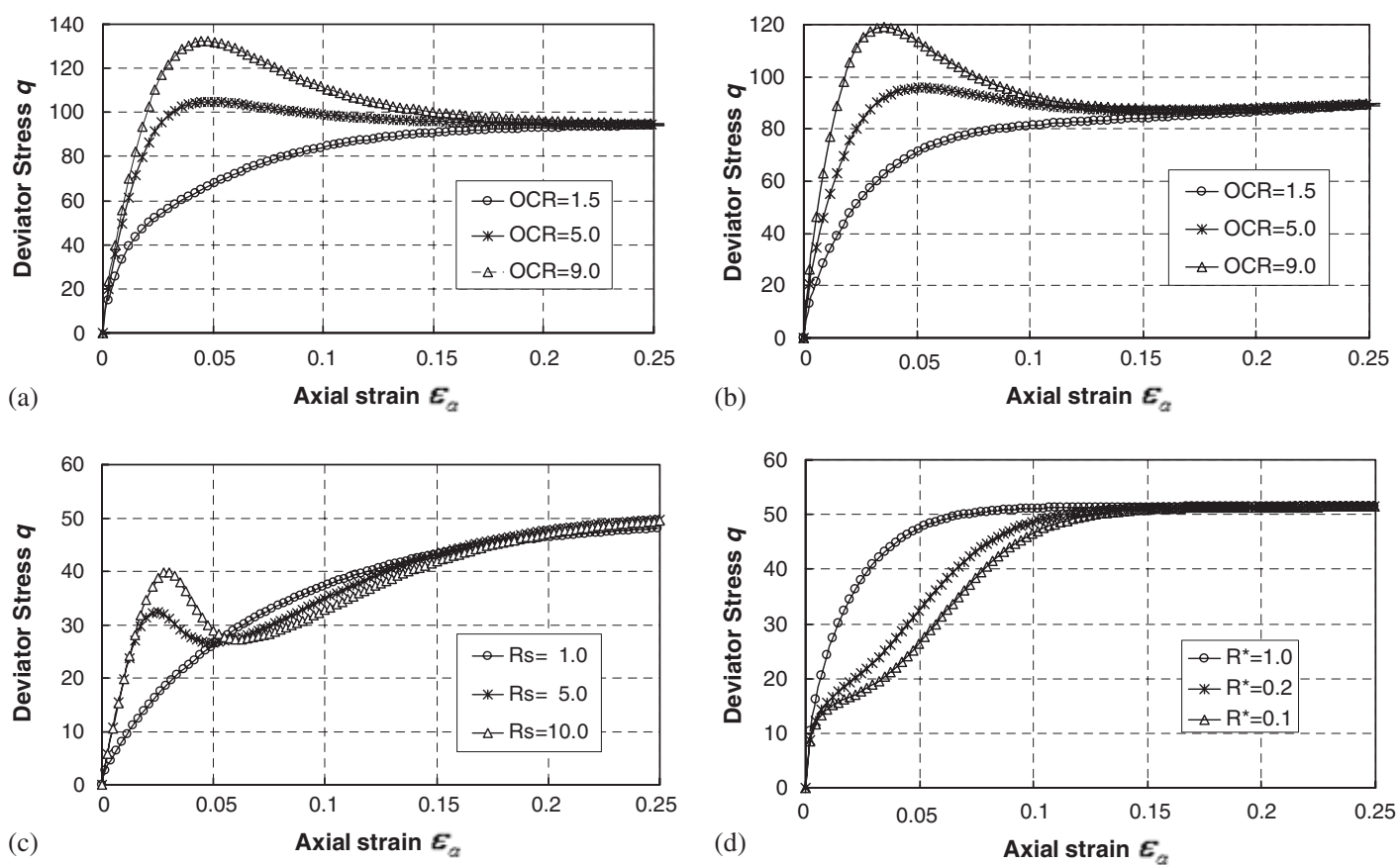

Figure 4. Sensitivity study to overconsolidation ratio and initial structure for the KHSM and SSLSM: (a) KHSM to OCR; (b) SSLSM to OCR; (c) KHSM to initial structure; and (d) SSLSM to initial structure.

the state of structure the higher the peak shear stress obtained. The reason for this difference in the SSLSM lies in that, even for the same soil, different initial structures imply different initial conditions (such as initial stress field and initial void ratio). In general, the compression line for structured soils is higher than the NCL for reconstituted soils. Thus, if the initial stresses are kept the same, larger initial void ratio is required for higher initial structure. Various approaches (such as curve fitting of the real stress-strain relation) are recommended by Asaoka et al. [37] to attain these initial conditions. However, it is difficult to do so due to the lack of experimental data. Therefore, in the computations for the SSLSM, the initial conditions for all structured soils are assumed to be the same for the sake of simplicity. Nevertheless, the simulations clearly show the initial structure does have a significant influence on the mechanic response of both the KHSM and SSLSM.

4.1.2. Numerical performance. The numerical performance of the stress integration scheme is studied here using the KHSM with initial conditions and material properties given in Table II $\left(R_{\mathrm{S}}=5.0\right.$ and OCR $\left.=4.0\right)$. The influence of the prescribed error tolerance is first investigated. Here, we assumed the yield surface tolerance (FTOL) is fixed at $10^{-9}$ and the displacement error tolerance is fixed at $10^{-4}$. The stress tolerance (STOL) varies from $10^{-3}$ to $10^{-9}$. The load is applied in form of prescribed axial strain and a total axial strain of $10 \%$ is imposed in 20 coarse increments. The obtained stress-strain curves are shown in Figure 5, which confirms that the variation of the tolerance does not cause any significant difference in the predicted local stressstrain results. Figure 5(b) plots the relative error in the vertical stress against the axial strain. 

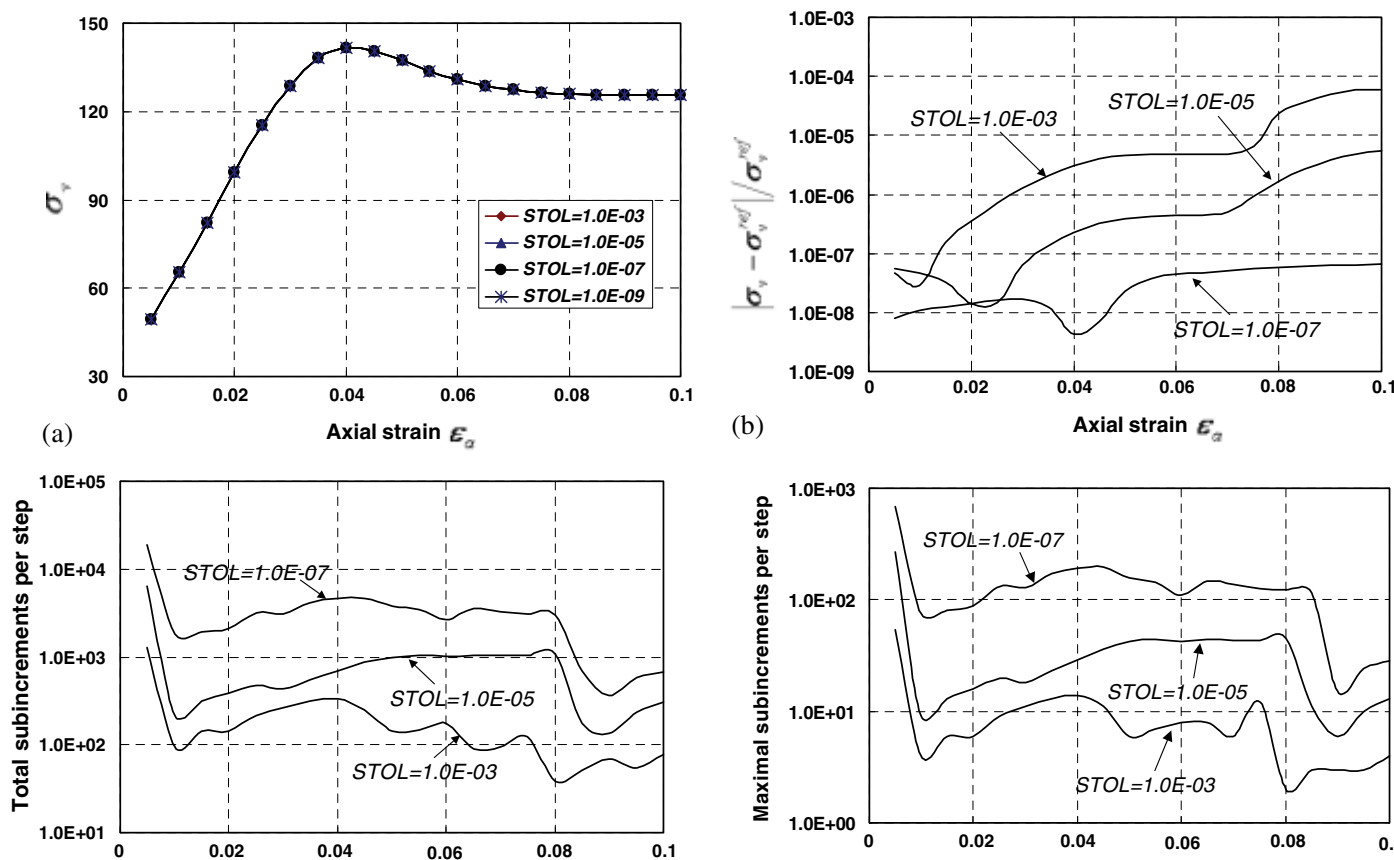

(c)

Axial strain $\varepsilon$

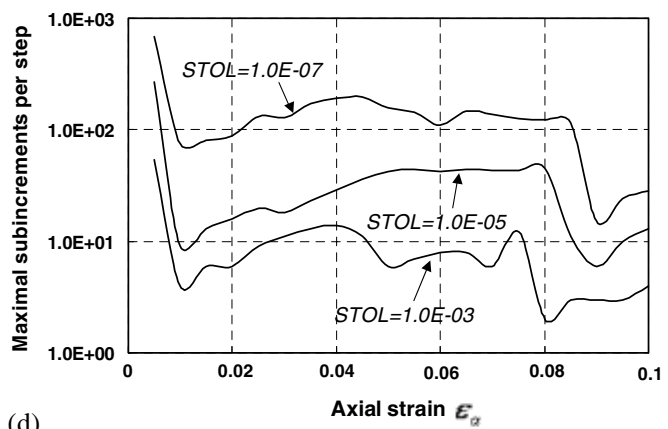

Figure 5. Models response and subincrements for KHSM under different prescribed tolerances: (a) stressstrain curves; (b) stress errors; (c) total subincrements per step; and (d) maximal subincrements per step.

The reference vertical stress $\sigma_{\mathrm{v}}^{\text {ref }}$ is obtained using a STOL $=10^{-9}$ and 10000 load increments. As may be observed, the relative error is well controlled under each prescribed tolerance. Figure 5(c) and (d) show the total and the maximum subincrements used at each load increment, respectively. An increase of the stress error tolerance by two orders (100) results in a decrease in the number of subincrements roughly by 10 times. Therefore, the increase of subincrements in cases of smaller tolerance is affordable for the computation. Actually, this is further confirmed by the CPU times used for each case. The total CPU times used for the four cases of STOL $=$ $10^{-3}, 10^{-5}, 10^{-7}, 10^{-9}$, are $3,7,16$ and $45 \mathrm{~s}$, respectively. Only a marginal increase in CPU times is resulted in when the stress tolerance is tightened. In Figure 5(c) and (d), we also note a significant number of subincrements have been used in the first load increment, whereas the stress error (Figure 5(b)) is relatively small. This type of behaviour implies that the subincrementation is actually controlled by the error in the hardening parameters, which is not shown here. For the KHSM model, the elastic region is very small and plastic yielding starts right after loading. Because the centre of the bubble yield surface is initially located on the $p^{\prime}$ axis and $q_{\bar{\alpha}}=0$, the relative error in the hardening parameter defined by Equation (23) is then very large, even the absolute error is very small. This type of behaviour always occurs when the denominator in the relative error is zero (for example a problem with a zero initial stress), and can be avoided by using a combined error measure where the absolute error replaces the relative error when the denominator is too small. 


\subsection{Rigid strip footing}

Analysis of a rigid strip footing can be a difficult numerical exercise due to the singularity at the edge of the footing and the strong rotation of the principal stresses. The KHSM and the SSLSM, as well as the MCC model, are used to simulate the soil behaviour. Material parameters for these models are listed in Table III. Note that these parameters are selected so that the model responses from both the KHSM and the SSLSM coincide with that from the MCC model when there is no initial structure in the soil. The critical state void ratio at $p^{\prime}=1$ is assumed to have a homogeneous value of 1.8 over the soil depth. The soil is assumed to be overconsolidated to $15 \mathrm{kPa}$ at ground surface. The footing geometry and the finite element mesh are shown in Figure 6 , with $L / 2=1$. The domain is divided into 288 triangular six-noded elements with a total of 1143 degrees of freedom. The left and right boundaries are fixed in the horizontal direction but allowed to move in the vertical direction, whereas the bottom boundary is locked in both directions. A prescribed displacement of $0.2 \mathrm{~B}$ is applied to the nodes under the footing in 50 coarse increments, and an equivalent footing load is found by summing the appropriate nodal reactions. Initial structures are attributed to the KHSM and SSLSM. The displacement tolerance (DTOL) is set to $10^{-3}$, while the stress integration tolerance (STOL) and the yield surface tolerance (FTOL) are set to $10^{-6}$ and $10^{-9}$, respectively.

Figure 7 illustrates the load-displacement curves obtained by the KHSM and the SSLSM in comparison with the MCC model. The effects of the initial structure of the soil on the loaddisplacement curves are apparent for both two structure models (Figure 7(a)). As may be observed, curves from both KHSM and SSLSM exhibit an obvious peak before they fall into the curve of MCCM's. This peak occurs at around 0.93 footing displacement for the SSLSM and 0.19 for the KHSM. Figure 7(b) and (c) further depict the evolution of structural index at reference point $a, b, c$ and $d$ (as shown in Figure 6) for the KHSM and SSLSM. It is readily to find the shallower the point is, the quicker the initial structure decays, which is a direct consequence of the plastic strain development in the simulated domain. It can also be noted that the destructuration in the KHSM starts at the beginning of the loading (Figure 7(b)), the destructuration in the SSLSM is somewhat delayed (Figure 7(c)). It is further noted that the destructuration at point $a$ in the SSLSM roughly coincides with the peak footing load in Figure 7(a). With the adopted model parameters, the elastic region of the KHSM is so small (as $r_{\mathrm{b}}$ ) that plastic yielding immediately starts upon loading, and thus the impact of destructuration on

Table III. Model parameter selection for footing problem.

\begin{tabular}{lccc}
\hline Models & KHSM & SSLSM & MCC \\
\hline Compression index & $\lambda^{*}=0.11$ & $\lambda=0.25$ & $\lambda=0.25$ \\
Swelling index & $k^{*}=0.0131$ & $k=0.052$ & $k=0.052$ \\
Poisson's ratio & $\mu=0.3$ & $\mu=0.3$ & $\mu=0.3$ \\
Density & $\gamma=6 \mathrm{kN} / \mathrm{m}^{3}$ & $\gamma=6 \mathrm{kN} / \mathrm{m}^{3}$ & $\gamma=6 \mathrm{kN} / \mathrm{m}^{3}$ \\
Frictional angle & $\phi=23^{\circ}$ & $\phi=23^{\circ}$ & $\phi=23^{\circ}$ \\
Slope of CSL & $M_{\max }=0.9$ & $M_{\max }=0.9$ & $M_{\max }=0.9$ \\
Other specific & $\omega=3.96, B=3.52$, & $m=9.64, a=0.51$ & $e_{0}=1.6$ \\
parameters & $\psi=1.53, r_{\mathrm{b}}=0.05$, & $A_{\mathrm{d}}=0.45, \alpha=0.77$, & \\
& $A_{\mathrm{d}}=0.55, \alpha=0.77$, & $R^{*}=0.6, e_{0}=2.35$ & \\
& $R_{\mathrm{s}}=5.4, e_{0}=1.6$ & & \\
\hline
\end{tabular}




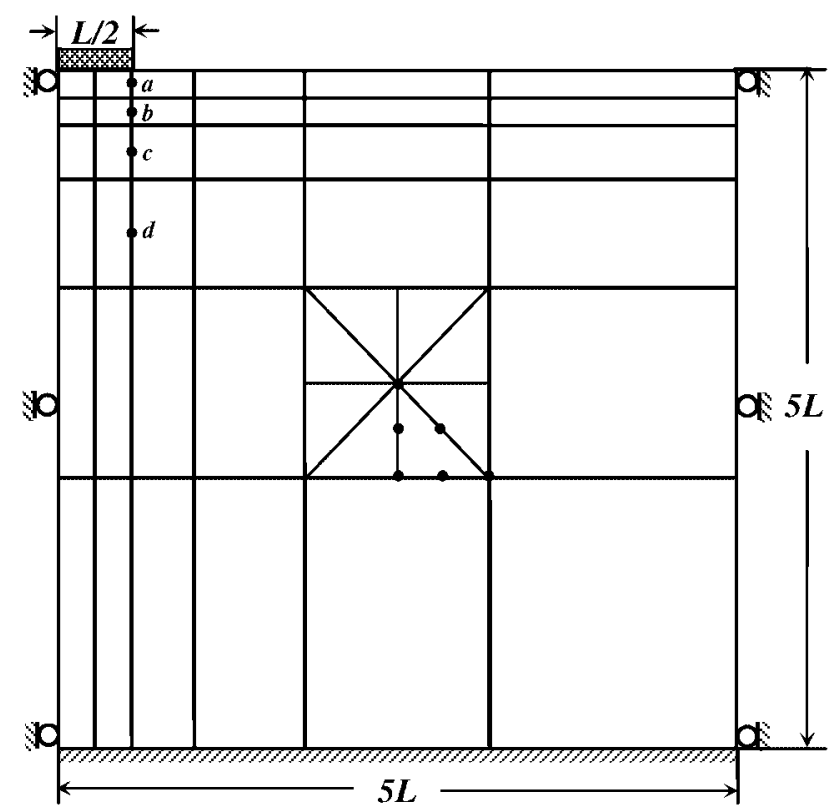

Figure 6. Mesh for rigid strip footing: 288 triangular six-noded elements with 625 nodes and 1143 degrees of freedom.

the overall mechanical response is also reflected from the beginning. In the SSLSM, the elastic region is relatively large, and the material behaves elastically until roughly the peak load. However, once plastic yielding occurs, the initial structural will decay rapidly and its influence on the overall mechanical response then becomes visible.

The numerical performance of the implementation of the two complex models is further investigated for this boundary value problem. The displacement tolerance (DTOL) is fixed at $10^{-3}$, and the yield surface tolerance at $10^{-9}$. The stress tolerance (STOL) varies between $10^{-4}$ and $10^{-7}$. Table IV presents the overall CPU times, the total successful subincrements at all integration points per load increment, the maximum successful subincrements amongst the integration points per load increment, and the relative stress error for the three models of MCCM, KHSM and SSLSM. The overall CPU times do not increase significantly as the STOL becomes more stringent. The total numbers of successful subincrements and the numbers of maximum subincrements increase roughly by a factor of $\sqrt{10}$ when the stress tolerance is tightened by a factor of 10 . The relative stress errors for the three models are all controlled under the stress tolerance. In computing the relative stress error, a reference solution obtained with a STOL $=10^{-9}$ is used. Another observation is that the KHSM uses most subincrements among the three models. The reason for this is that the KHSM has the most number of hardening parameters.

Figure 8 depicts the total number of successful subincrements used in each load increment for the three models. As is shown, a higher resolution of the stress tolerance will always invoke a larger number of successful subincrements during each loading step for all the three models. The number of the successful subincrements increases again roughly $\sqrt{10}$ times as the stress tolerance is tightened by a factor of 10. As is observed from Figure 8(a) and (c), the MCCM and 

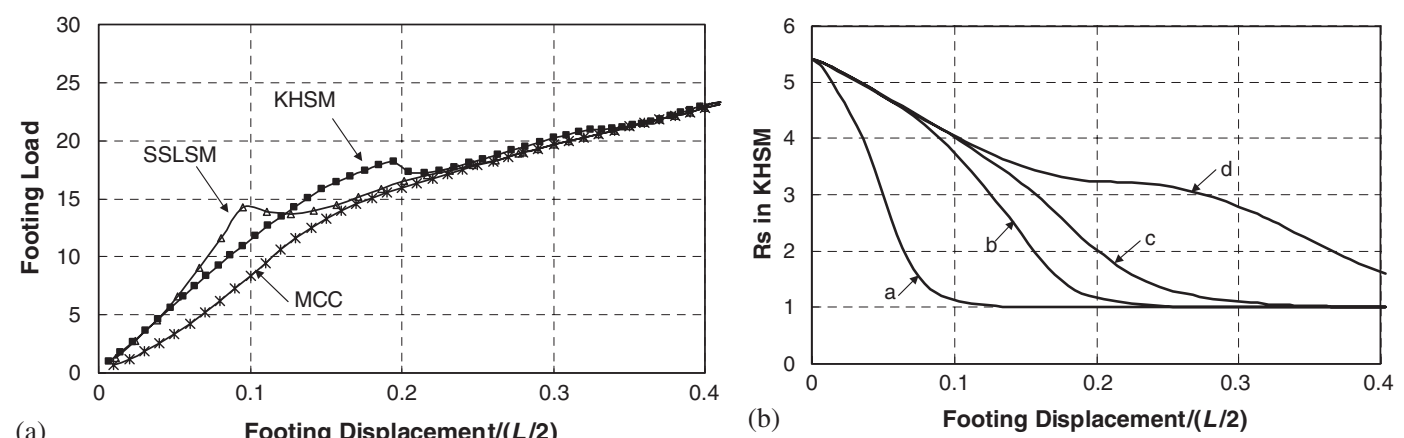

(a)

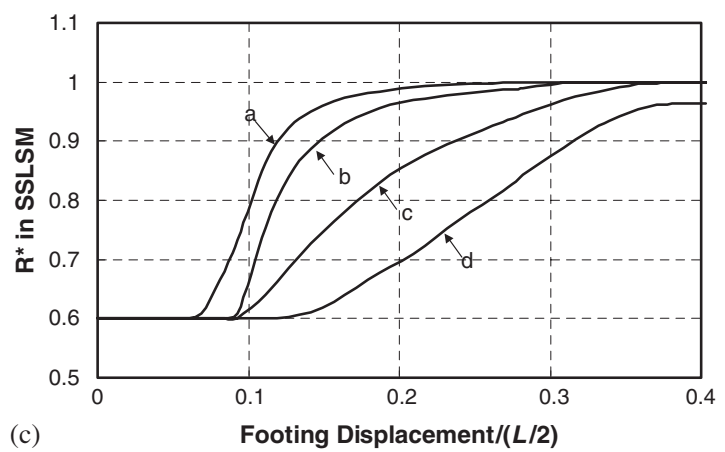

Figure 7. The rigid footing problem analysed by the MCCM, KHSM and SSLSM: (a) footing displacement vs footing load; (b) footing displacement vs structural index $R_{\mathrm{s}}$ evolution in KHSM at reference points; and (c) footing displacement vs structural index $R^{*}$ evolution in SSLSM at reference points.

Table IV. Rigid footing on KHSM by explicit Euler substepping integration.

\begin{tabular}{lccccc}
\hline Model & Stress tolerance & $\begin{array}{c}\text { CPU } \\
\text { time (s) }\end{array}$ & $\begin{array}{c}\text { Total successful } \\
\text { subincrements }\end{array}$ & $\begin{array}{c}\text { Max. successful } \\
\text { subincrements }\end{array}$ & $\begin{array}{c}\text { Relative } \\
\text { stress error }\end{array}$ \\
\hline MCC & STOL $=1.0 \mathrm{E}-4$ & 19 & 8118 & 17 & $3.890 \mathrm{E}-05$ \\
& STOL $=1.0 \mathrm{E}-5$ & 19 & 21019 & 44 & $9.205 \mathrm{E}-06$ \\
& STOL $=1.0 \mathrm{E}-6$ & 21 & 61823 & 120 & $6.845 \mathrm{E}-07$ \\
& STOL $=1.0 \mathrm{E}-7$ & 31 & 190824 & 437 & - \\
KHSM & STOL $=1.0 \mathrm{E}-4$ & 23 & 28827 & 71 & $7.860 \mathrm{E}-05$ \\
& STOL $=1.0 \mathrm{E}-5$ & 24 & 64101 & 126 & $1.780 \mathrm{E}-06$ \\
& STOL $=1.0 \mathrm{E}-6$ & 30 & 178601 & 244 & $2.729 \mathrm{E}-07$ \\
& STOL $=1.0 \mathrm{E}-7$ & 48 & 542249 & 608 & - \\
& STOL $=1.0 \mathrm{E}-4$ & 19 & 9654 & 18 & $8.200 \mathrm{E}-05$ \\
& STOL $=1.0 \mathrm{E}-5$ & 19 & 24589 & 50 & $5.520 \mathrm{E}-06$ \\
& STOL $=1.0 \mathrm{E}-6$ & 21 & 68181 & 154 & $5.783 \mathrm{E}-07$ \\
& STOL $=1.0 \mathrm{E}-7$ & 37 & 210311 & 484 & - \\
\hline
\end{tabular}

SSLSM do not require any subincrement initially, because of the elastic regions in the model. For the KHSM, the elastic region is very small and plastic yielding occurs very early, and thus subincrementation is required right from the beginning. 


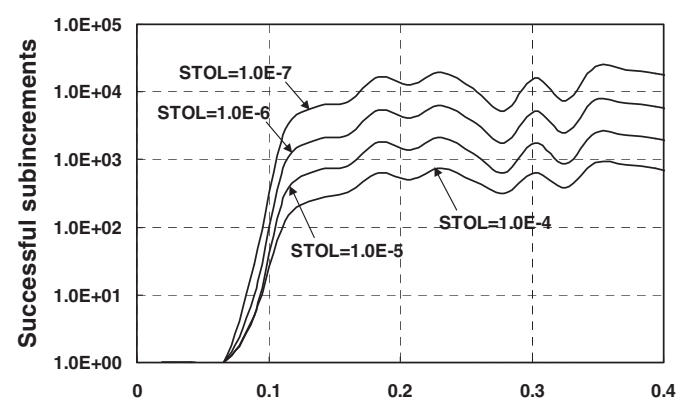

(a)
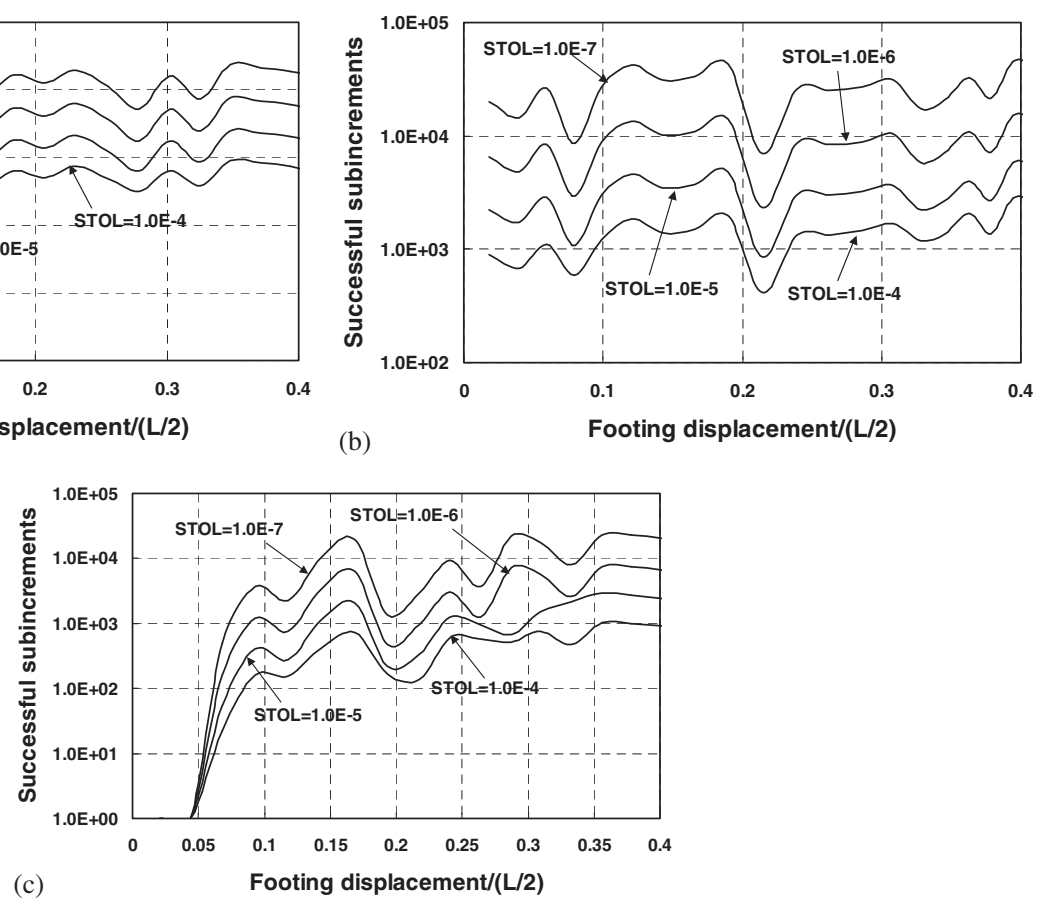

Figure 8. Total number of successful subincrements used during the loading course:

(a) MCC; (b) KHSM; and (c) SSLSM.

\section{CONCLUSIONS}

Some key conclusions from this study are as follows.

(1) The explicit stress integration scheme with automatic substepping and error control can be used to integrate advanced soil models with multiple yield surfaces and complex hardening laws. This has been tested with two critical-state models- the KHSM and the SSLSM - that take into account the effects of the initial structure and destructuration. This scheme is particularly effective in handling multiple hardening parameters along with complicated hardening laws. No specific measure is required to handle the bounding surface. When the stress integration scheme is used in association with an automatic load stepping scheme, the small-strain non-linearity in the KHSM and SSLSM can be well simulated. No numerical problems were encountered with the automatic substepping scheme and load stepping schemes for both drained triaxial compression tests and a rigid strip footing problem.

(2) It is illustrated the explicit integration scheme remains to be efficient, accurate and robust. For both the drained triaxial problem and the rigid footing problem, the increase in the computational effort is relatively affordable when the tolerance is substantially tightened. The number of the successful subincrements increases roughly $\sqrt{10}$ times as the stress tolerance is tightened by a factor of 10 . The presence of additional hardening parameters in the complex models may result in larger relative error when some of hardening parameters are initially zero, which may in turn lead to a larger number of subincrements. 
(3) The implementation of these complex soil models into finite element codes facilitates their application into boundary-value problems. Modelling of overconsolidated soils under drained triaxial compression shows that both the KHSM and the SSLSM predict a smoother stress-strain curve than those from standard Cam-clay models. Simulation of structured soils under drained triaxial compression indicates that the initial structure has a significant effect on the predictions of both the KHSM and the SSLSM. Both models predict that a soil with an initial structure can sustain a larger shear stress than the remoulded soil. However, to attain this by the SSLSM, special attention must be paid to ensure that the initial void ratio corresponds to the initial structure. Modelling of the rigid strip footing by the KHSM and the SSLSM illustrates that implemented complex soil models can be applied to solve boundary-value problems. The effects of the initial structure on the load-displacement response are noticeable when the predictions of these models are compared that of the modified Cam-clay model.

\section{APPENDIX A}

For the KHSM, the following expressions for $\mathbf{B}$ can be obtained:

$$
\begin{aligned}
B_{1} & =\frac{p_{\mathrm{c}}^{\prime}}{\lambda^{*}-k^{*}} \frac{\partial f_{\mathrm{b}}}{\partial p^{\prime}} \\
B_{2} & =\frac{\omega\left(1-R_{\mathrm{s}}\right)}{\lambda^{*}-k^{*}} \mathrm{~d} \varepsilon_{\mathrm{d}} \\
\left(B_{3}, B_{4}\right)^{\mathrm{T}} & =\bar{\alpha}\left(\frac{B_{2}}{R_{\mathrm{s}}}+\frac{B_{2}}{p_{\mathrm{c}}^{\prime}}\right)+B_{\mu}\left(\boldsymbol{\sigma}_{\mathrm{c}}-\boldsymbol{\sigma}\right)
\end{aligned}
$$

where

$$
\begin{aligned}
B_{\mu} & =\left\{H-\mathbf{n}:\left[\boldsymbol{\sigma} \frac{\mathrm{d} p^{\prime}}{p_{\mathrm{c}}^{\prime}}+\bar{\alpha} \frac{\mathrm{d} R_{\mathrm{s}}}{R_{\mathrm{s}}}\right]\right\} / b, \quad \mathbf{n}=\left(\frac{\partial f_{\mathrm{b}}}{\partial p^{\prime}}, \frac{\partial f_{\mathrm{b}}}{\partial q}\right)^{\mathrm{T}}, \quad \boldsymbol{\sigma}=\left(p^{\prime}, q\right)^{\mathrm{T}} \\
\mathrm{d} \varepsilon_{\mathrm{d}} & =\sqrt{\left(1-A_{\mathrm{d}}\right)\left(\frac{\partial f_{\mathrm{b}}}{\partial p^{\prime}}\right)^{2}+A_{\mathrm{d}}\left(\frac{\partial f_{\mathrm{b}}}{\partial q}\right)^{2}}, \quad \overline{\boldsymbol{\alpha}}=\left(p_{\bar{\alpha}}^{\prime}, q_{\bar{\alpha}}\right)^{\mathrm{T}}, \quad b=\mathbf{n} \cdot\left(\boldsymbol{\sigma}_{\mathrm{c}}-\boldsymbol{\sigma}\right) \\
\boldsymbol{\sigma}_{\mathrm{c}} & =\left(R_{\mathrm{s}} p_{\mathrm{c}}^{\prime}+\frac{R_{\mathrm{s}}}{R_{\mathrm{b}}}\left(p^{\prime}-p_{\bar{\alpha}}^{\prime}\right), \frac{R_{\mathrm{s}}}{R_{\mathrm{b}}}\left(q-q_{\bar{\alpha}}\right)\right)^{\mathrm{T}}, \quad b_{\max }=2 \frac{\left(R_{\mathrm{s}}-R_{\mathrm{b}}\right)}{R_{\mathrm{b}}} \mathbf{n} \cdot(\boldsymbol{\sigma}-\bar{\alpha}) \\
H & =\frac{B p_{\mathrm{c}}}{\left(\lambda^{*}-k^{*}\right) R_{\mathrm{b}}}\left(\frac{b}{b_{\mathrm{max}}}\right)^{\psi}+H^{\mathrm{c}}, \quad H^{\mathrm{c}}=\frac{R_{\mathrm{s}} p_{\mathrm{c}}^{\prime}\left\{\xi\left[\left(p^{\prime}-p_{\bar{\alpha}}^{\prime}\right)+R_{\mathrm{b}} p_{\mathrm{c}}^{\prime}\right]\right\}}{\left(\lambda^{*}-k^{*}\right)\left[\left(p^{\prime}-p_{\bar{\alpha}}^{\prime}\right)^{2}+\left(\left(q-q_{\bar{\alpha}}\right) / M^{2}\right)^{2}\right]} \\
\xi & =\left(p^{\prime}-p_{\bar{\alpha}}^{\prime}\right)+\frac{\omega\left(1-R_{\mathrm{s}}\right)}{R_{\mathrm{s}}} \mathrm{d} \varepsilon_{\mathrm{d}}
\end{aligned}
$$

and $\omega, A_{\mathrm{d}}, B$, and $\psi$ are additional model parameters.

In the SSLSM, B has the following elements:

$$
B_{1}=\frac{v \tilde{p}_{\mathrm{c}}^{\prime}}{\lambda-k} \frac{\partial f_{\mathrm{sub}}}{\partial p^{\prime}}, \quad B_{2}=-\frac{v M}{\lambda-k} m \ln R \mathrm{~d} \varepsilon_{\mathrm{d}}, \quad B_{3}=\frac{v M a}{\lambda-k} R^{*}\left(1-R^{*}\right) \mathrm{d} \varepsilon_{\mathrm{d}}
$$


where

$$
\mathrm{d} \varepsilon_{\mathrm{d}}=\sqrt{\left(1-A_{\mathrm{d}}\right)\left(\frac{\partial f_{\mathrm{sub}}}{\partial p^{\prime}}\right)^{2}+A_{\mathrm{d}}\left(\frac{\partial f_{\mathrm{sub}}}{\partial q}\right)^{2}}
$$

and $m, a$, and $A_{\mathrm{d}}$ are additional model parameters. $\lambda$ and $k$ are the slopes of the normal compression line and swelling line in the plane of $v-\ln p^{\prime}$, respectively.

\section{ACKNOWLEDGEMENTS}

The authors would thank Prof. David Potts for his helpful suggestions. Comments from the two anonymous reviewers are also gratefully acknowledged.

\section{REFERENCES}

1. Roscoe KH, Schofield AN, Wroth CP. On the yielding of soils. Géotechnique 1958; 8:22-52.

2. Roscoe KH, Schofield AN. Mechanical behaviour of an idealised 'wet' clay. Proceeding of 2nd European Conference on Soil Mechanics and Foundation Engineering, vol. 1, Wiesbaden, 1963; 47-54.

3. Schofield AN, Wroth CP. Critical State Soil Mechanics. McGraw-Hill: London, 1968.

4. Roscoe KH, Burland JB. On the generalised stress-strain behaviour of 'wet' clay. Engineering Plasticity. Cambridge University Press: Cambridge, 1968; 535-560.

5. Carter JP, Booker JR, Wroth CP. A critical state soil model for cyclic loading. In Soil Mechanics-Transient and Cyclic Loads, Pande GN, Zienkiewicz OC (eds). Wiley: New York, 1982; 219-252.

6. Muir Wood D. Kinematic hardening model for structured soil. In Numerical Models in Geomechanics, Pande NV, Pietruszczak S (eds). Balkema: Rotterdam, 1995; 83-88.

7. Sheng DC, Sloan SW, Yu HS. Aspects of finite element implementation of critical state models. Computational Mechanics 2000; 26:185-196.

8. Liu MD, Carter JP. On the volumetric deformation of reconstituted soils. International Journal for Numerical and Analytical Methods in Geomechanics 2000; 24:101-133.

9. Rouainia M, Muir Wood D. A kinematic hardening constitutive model for natural clays with loss of structure. Géotechnique 2000; 50(2):153-164.

10. Asaoka A. Consolidation of clay and compaction of sand-an elasto-plastic description. The 12th Asian Regional Conference on SMGE, Singapore, August 2003.

11. Simo JC, Taylor RL. Consistent tangent operators for rate-independent elasto-plasticity. Computer Methods in Applied Mechanics and Engineering 1985; 48:101-118.

12. Potts DM, Gens A. A critical assessment of methods of correcting for drift from the yield surface in elastoplastic finite element analysis. International Journal for Numerical and Analytical Methods in Geomechanics 1985; 9:149-159.

13. Oritz M, Simo JC. An analysis of a new class of integration algorithms for elasto-plastic constitutive relations. International Journal for Numerical Methods in Engineering 1986; 23:353-366.

14. Britto AM, Gunn MJ. Critical State Soil Mechanics via Finite Elements. Ellis Horwood: Chichester, 1987.

15. Gens A, Potts DM. Critical state models in computational geomechanics. Engineering Computations 1988; 5: 178-197.

16. Borja RI, Lee SR. Cam-clay plasticity. Part I: implicit integration of elasto-plastic constitutive relations. Computer Methods in Applied Mechanics and Engineering 1990; 78:49-72.

17. Borja RI. Cam-clay plasticity. Part II: implicit integration of constitutive equations based on a non-linear elastic stress predictor. Computer Methods in Applied Mechanics and Engineering 1991; 88:225-240.

18. Jeremic B, Sture S. Implicit integration in elastoplastic geotechnics. Mechanics of Cohesive-Frictional Materials 1997; 2:165-183.

19. Macari EJ, Weihe S, Arduino P. Implicit integration of elastoplastic constitutive models for frictional materials with highly non-linear hardening functions. Mechanics of Cohesive-Frictional Materials 1997; 2:1-29.

20. Borja RI, Lin CH, Montáns FJ. Cam-clay plasticity. Part IV: implicit integration of anisotropic bounding surface model with nonlinear hyperelasticity and ellipsoidal loading function. Computer Methods in Applied Mechanics and Engineering 2001; 190:3293-3323.

21. Tamagnini C, Castellanza R, Nova R. A generalized backward Euler algorithm for the numerical integration of an isotropic hardening elastoplastic model for mechanical and chemical degradation of bonded geomaterials. International Journal for Numerical and Analytical Methods in Geomechanics 2002; 26:963-1004. 
22. Borja RI, Sama KM, Sanz PF. On the numerical integration of three-invariant elastoplastic constitutive models. Computer Methods in Applied Mechanics and Engineering 2003; 192:1227-1258.

23. Wissmann JW, Hauck C. Efficient elastic-plastic finite element analysis with higher order stress point algorithms. Computers and Structures 1983; 17:89-95.

24. Sloan SW. Substepping schemes for the numerical integration of elastoplastic stress-strain relations. International Journal for Numerical Methods in Engineering 1987; 24:893-911.

25. Hashash YMA, Whittle AJ. Integration of the modified Cam-clay model in nonlinear finite element analysis. Computers and Geotechnics 1992; 14:59-83.

26. Sloan SW. Substepping schemes for the numerical integration of elastoplastic stress-strain relations. International Journal for Numerical Methods in Engineering 1987; 24:893-911.

27. Sloan SW, Abbo AJ, Sheng DC. Refined explicit integration of elastoplastic models with automatic error control. Engineering Computations 2001; 18(1/2):121-154.

28. Abbo AJ, Sloan SW. An automatic load stepping algorithm with error control. International Journal for Numerical Methods in Engineering 1996; 39:1737-1759.

29. Sheng DC, Sloan SW. Loading stepping schemes for critical state models. International Journal for Numerical Methods in Engineering 2001; 50:67-93.

30. Luccioni LX, Pestana JM, Taylor RL. Finite element implementation of non-linear elastoplastic constitutive laws using local and global explicit algorithms with automatic error control. International Journal for Numerical Methods in Engineering 2001; 50:1191-1212.

31. Potts DM, Ganendra D. An evaluation of substepping and implicit stress point algorithms. Computer Methods in Applied Mechanics and Engineering 1994; 119:341-354.

32. Crisfield MA. Nonlinear Finite Element Analysis of Solids and Structures, vol. 1: Essentials. Wiley: Chichester, 1991.

33. Crisfield MA. Nonlinear Finite Element Analysis of Solids and Structures, vol. 2. Wiley: Chichester, 1997.

34. Al-Tabbaa A, Muir Wood D. An experimentally based 'bubble' model for clay. In Proceedings of 3rd International Conference on Numerical Models in Geomechanics NUMOG III, Pietruszczak S, Pande GN (eds). Elsevier Applied Sciences: Amsterdam, 1989; 91-99.

35. Hashiguchi K. Subloading surface model in unconventional plasticity. International Journal of Solids and Structures 1989; 25:917-945.

36. Dafalias YF. Bounding surface plasticity. I: mathematical foundation and hypoelasticity. Journal of Engineering Mechanics (ASCE) 1986; 112(EM9):966-987.

37. Asaoka A, Nakano M, Noda T. Superloading yield surface concept for highly structured soil behaviour. Soils and Foundations 2000; 40(2):99-110. 